\title{
Fatores associados a sintomas de transtornos alimentares entre escolares da rede pública da cidade do Salvador, Bahia
}

Factors associated with symptoms of eating disorders among students from public schools in Salvador, Bahia

Thaisy Cristina Honorato Santos Alves', Mônica Leila Portela de Santana', Rita de Cássia Ribeiro Silva', Elizabete de Jesus Pinto ${ }^{1}$, Ana Marlúcia Oliveira Assis

\section{RESUMO}

Objetivo: Identificar prevalência e fatores associados aos sintomas de transtornos alimentares entre escolares. Métodos: Estudo transversal envolvendo 365 escolares, de 7 a 14 anos de idade, do ensino fundamental de Salvador/BA. Aplicou-se nos escolares $\leq 12$ anos o Teste de Comportamentos Alimentares e Imagem Corporal e, naqueles $\geq 13$ anos de idade, o Teste de Atitudes Alimentares-26, o Teste de Investigação Bulímica de Edinburgh e o Teste de Imagem Corporal. O Child Behavior Checklist foi aplicado para todos os escolares. Coletaram-se dados antropométricos e alimentares, sobre estilo de vida, demográficos, socioeconômicos e puberais. Na análise estatística utilizou-se regressão de Poisson. Resultados: Sintomas de transtornos alimentares estavam presentes em $23 \%$ dos escolares. Idade (RP: 1,25; IC95\%: 1,11-1,40), insatisfação com imagem corporal (RP: 4,23; IC95\%: 2,53-7,08), problema de comportamento de internalização (RP: 1,78; IC95\%: 1,11-2,85), substituição das refeições por consumo de balas (RP: 2,14; IC95\%: 1,24-3,69), maior consumo de frutas e outros vegetais (RP: 2,49; IC95\%: 1,55-3,99) e escolaridade materna de $5^{a}$ a $8^{a}$ série (RP: 1,95; IC95\%: 1,06-3,58) associaram-se ao aumento da ocorrência dos sintomas de transtornos alimentares. Conclusão: Observou-se alta prevalência de sintomas de transtornos alimentares entre os escolares; fatores sociodemográficos e comportamentais estavam associados ao evento.

\section{ABSTRACT}

Objective: To identify the prevalence and factors associated with symptoms of eating disorders among students. Methods: Cross sectional study involving 365 students from 7 to 14 years old from elementary school in Salvador, Bahia. It was applied for the students of $<12$ years old the Eating Behaviors and Body Image Test and for those of $>13$ years old it was adopted the Eating Attitudes Test-26, Bulimic Investigatory Test of Edinburgh and Body Shape Questionnaire. The Child Behavior Checklist was administered to all students. Data was collected anthropometric and dietary, lifestyle, demographic, socioeconomic, and

1 Universidade Federal da Bahia (UFBA) - Escola de Nutrição.

Recebido em

22/8/2011

Aprovado em $06 / 3 / 2012$
Endereço para correspondência: Thaisy Cristina Honorato Santos Alves

Universidade Federal da Bahia - Escola de Nutrição - Núcleo de Nutrição e Epidemiologia

Rua Araújo Pinho, 32, Canela - 40110-150 - Salvador, BA

Telefones: (71) 3283-7700/9111-9900

E-mail: thaisyhonorato@yahoo.com.br 


\section{Keywords}

Eating disorders, anorexia nervosa, bulimia nervosa, students.

\section{INTRODUÇÃO}

Transtornos alimentares constituem quadros psiquiátricos que podem estar associados à morbimortalidade, podendo ocasionar desde prejuízos emocionais e sociais até consequências fisiopatológicas relacionados aos sistemas metabólico e endócrino'. Dentre os transtornos alimentares mais conhecidos, destacam-se a anorexia e a bulimia nervosa.

A anorexia nervosa é caracterizada pelo temor intenso de ganho ponderal, superestimação na percepção da forma ou tamanho do corpo e imposição de autorrestrição ao consumo alimentar ${ }^{2}$. A bulimia nervosa caracteriza-se por episódios recorrentes de voracidade alimentar, seguidos por condutas purgativas (autoindução de vômitos ou uso indiscriminado de laxantes, diuréticos, inibidores de apetite ou enemas) ou não purgativas (jejuns e exercícios físicos excessivos) 3 .

Evidências científicas indicam que a etiologia dos transtornos alimentares seja multifatorial. Acredita-se que a adolescência represente o ciclo da vida de maior exposição para sua ocorrência, embora atualmente esses distúrbios alimentares estejam iniciando em idade mais precoce da vida ${ }^{4,5}$.

A ocorrência acentuada dos transtornos alimentares na adolescência encontra explicação na busca da identidade pessoal e do senso de independência social e emocional nessa fase da vida. Sobressai, ainda, a influência dos fatores psicológicos, aliada ao forte apelo sociocultural do culto à magreza. Tais características podem se manifestar negativamente nas escolhas alimentares ${ }^{6}$.

Esse evento pode se associar, ainda, às comorbidades psiquiátricas, incluindo transtornos ansiosos, particularmente fobia social e transtorno obsessivo-compulsivo.

Estudos indicam que a ocorrência dos transtornos alimentares é mais elevada em mulheres, consequência da elevada cobrança social pela busca do corpo perfeito no sexo feminino ${ }^{8,9}$. Entretanto, o fato de os homens serem rotineiramente excluídos das amostras dos estudos que versam sobre esse objeto pode expressar-se na escassez de informações sobre esses transtornos no sexo masculino ${ }^{10}$.

O nível socioeconômico alto até recentemente era responsabilizado como importante fator de exposição para transtornos alimentares. Porém, informações atuais têm mostrado pouca ou nenhuma associação entre esses even$\operatorname{tos}^{11}$, permanecendo esse fator de exposição como uma questão não consensual até o momento.
A gravidade das consequências dos transtornos alimentares indica a pertinência de identificar precocemente os fatores que contribuem para a ocorrência de tais eventos, contribuindo para a adoção de medidas eficazes para assistência, controle e prevenção. Para tanto, o presente estudo visa identificar a prevalência e fatores associados aos sintomas de transtornos alimentares entre escolares da cidade de Salvador/BA.

\section{MÉTODOS}

Estudo de corte transversal, envolvendo escolares de 7 a 14 anos de idade, de ambos os sexos, matriculados na rede pública de ensino fundamental da cidade de Salvador.*

O processo amostral envolveu desenho complexo estruturado por conglomerado em três estágios: distritos sanitários, escolas e alunos.

Utilizaram-se as informações do banco de matrícula disponibilizado pelas Secretarias de Educação e Cultura do Município (SME) e de Educação do Estado da Bahia no ano de 2008. Por questões logísticas para equacionar a coleta de dados, selecionaram-se aleatoriamente 6 dos 12 distritos sanitários da cidade, para integrarem a amostra do estudo, comportando 117 escolas estaduais e 173 municipais. A amostra deste estudo foi composta por 365 escolares, constituindo subprojeto de uma investigação mais ampla que avaliou os fatores associados à anemia em escolares da cidade de Salvador ${ }^{12}$.

Considerando que a amostra de 365 escolares não foi estimada para avaliar a relação proposta neste estudo, decidiu-se por calcular o erro amostral a posteriori, para estimar a magnitude do erro com que esse número amostral permitiria avaliar a relação na amostra e a verdadeira relação na população. Nesse sentido, com base na prevalência de 23,0\% de sintomas de transtornos alimentares identificada entre os escolares, o número amostral previamente adotado permitiu determinar os fatores associados ao desfecho estudado com erro admissível de até 3\%, considerando o nível de confiança de 95\%.

\footnotetext{
* Constitui-se um subprojeto de um estudo mais amplo intitulado "Fatores psicossociais como elementos que repercutem nas condições de saúde, nutrição e desenvolvimento cognitivo de estudantes do ensino fundamental das escolas públicas de Salvador/BA", desenvolvido por docentes da Escola de Nutrição e do Instituto de Saúde Coletiva da Universidade Federal da Bahia.
} 
O estudo teve seu protocolo aprovado pelo Comitê de Ética do Instituto de Saúde Coletiva da Universidade Federal da Bahia. Após o conhecimento dos objetivos do estudo, os pais ou responsáveis pelo escolar que concordaram em participar assinaram o Termo de Consentimento Livre e Esclarecido; os analfabetos o fizeram por meio da impressão digital.

\section{Coleta de dados e variáveis}

A coleta de dados foi realizada entre março e dezembro de 2008. Os escolares foram selecionados aleatoriamente a partir da listagem dos alunos, por turma. Informações socioeconômicas e sobre comportamento do escolar foram fornecidas pelos responsáveis pelos participantes da pesquisa; os demais dados foram fornecidos pelos escolares e registrados em formulários apropriados.

A coleta dos dados foi realizada por pessoal qualificado e previamente treinado e obtida em três grandes blocos. O primeiro bloco compreendeu a identificação dos sintomas de transtornos alimentares. Para indivíduos de 7 a 12 anos de idade, utilizou-se o Teste de Comportamentos Alimentares e Imagem Corporal (EBBIT) 13,14. Para aqueles de 13 anos de idade ou mais, utilizaram-se o Teste de Atitudes Alimentares (EAT26) e o Teste de Investigação Bulímica de Edinburgh (BITE) ${ }^{15,16}$.

O EBBIT contém 42 itens de uma escala Likert de 0 a 4 pontos. Este estudo trabalhou com duas de suas três escalas: a da insatisfação com a imagem corporal e restrição alimentar e a de comportamentos de comer compulsivo ${ }^{13}$. Os escores médios obtidos pelo somatório dessas escalas foram comparados com a média e desvio-padrão de referência por idade. O ponto de corte utilizado para considerar o escolar como portador de sintomas de transtorno alimentar foi o somatório superior a tais valores de referência de acordo com a faixa etária ${ }^{14}$.

O EAT-26 mede comportamentos alimentares restritivos, como dieta e jejum, e comportamentos purgativos. Os 26 itens do EAT constituem uma escala Likert de 0 a 3 pontos. O ponto de corte maior que 21 pontos nesse instrumento identificou o indivíduo como em alto risco para sintoma de anorexia ${ }^{15,17}$.

O BITE identifica episódios bulímicos e fatores ligados à cognição e ao comportamento bulímico. Está dividido em duas subescalas que avaliam sintomas e gravidade. No presente estudo avaliou-se somente a subescala de sintomas. A resposta afirmativa a cada questão equivale a um ponto; menos de 10 pontos sugere comportamento normal; 10 a 19 demonstram comportamento pouco usual, porém não identifica diagnóstico de bulimia; 20 ou mais pontos indicam alto grau de desordem alimentar, presença de episódios bulímicos e possibilidade do diagnóstico de bulimia. O ponto de corte maior ou igual a 20 no BITE identificou o indivíduo como portador de sintoma de bulimia nervosa ${ }^{16,18}$

O segundo bloco destinou-se à avaliação da percepção da imagem corporal. Para escolares com idade igual ou menor que 12 anos, essa avaliação foi realizada pelo supramencionado EBBIT; para tanto, foram selecionadas as seis questões referentes à imagem corporal, considerando-se insatisfação sempre que pelo menos três das seis questões fossem afirmativas.

Para os maiores de 12 anos, utilizou-se o Teste de Imagem Corporal (BSQ) ${ }^{19}$, integrado por 34 itens em escala Likert de 0 a 6 pontos, para identificar insatisfação ou preocupação com peso e forma corporal nas quatro semanas anteriores à entrevista ${ }^{20,21}$. As categorias do BSQ foram determinadas a partir de escores, obtidos por meio do somatório dos pontos do instrumento assim discriminado: nenhuma preocupação: < ou igual a 80 pontos; preocupação leve: 81 a 110; preocupação moderada: 111 a 140; preocupação grave: > 140. Ponto de corte $>80$ identificou o indivíduo como portador de insatisfação com a imagem corporal.

O terceiro bloco foi constituído pelo Child Behavior Checklist (CBCL), destinado a identificar problemas de comportamento. $\mathrm{O}$ instrumento consiste em 118 itens que refletem problemas de comportamento e 20 de competência social. Neste estudo foram adotados os itens que identificam problemas de comportamento.

O CBCL contém oito escalas individuais referentes a problemas possivelmente presentes em crianças e adolescentes: ansiedade/depressão; isolamento; queixas somáticas; problemas de contato social; problemas com pensamento obsessivo ou distorcido; problemas de hiperatividade ou déficit de atenção; comportamento delinquente; comportamento agressivo; cuja soma dá origem à Escala Total de Problemas de Comportamento. Essa Escala Total é subdividida em Escala de Internalização, que corresponde às três primeiras escalas de problemas de comportamento, e Escala de Externalização, que corresponde às duas últimas escalas.

O CBCL é interpretado por meio da escala Likert de 0 a 2 pontos. A pontuação bruta obtida do somatório total é convertida em escores T por meio do software Assessment Data Manager (ADM)22. Em todas as escalas, o indivíduo é classificado como Clínico, Limítrofe ou Não Clínico. Neste estudo, optou-se pela junção dos casos limítrofes com os não clínicos. O indivíduo foi classificado como portador de possível problema comportamental quando a soma da pontuação dos escores $T$ foi maior do que $63^{22,23}$.

As informações antropométricas neste estudo foram compostas por peso e altura. O peso foi obtido por meio da balança digital portátil Master (Marte Balanças e Aparelhos de Precisão Ltda., São Paulo, Brasil), com capacidade para 199,95 kg e precisão de 50 g, admitindo-se variação máxima de $0,1 \mathrm{~kg}$ para medida de peso. O escolar usava roupas em tecidos leves. A estatura foi obtida por meio do estadiômetro portátil, da marca Leicester Height Measure (Leicester Height Measure, Londres, Inglaterra), com a leitura registrada no milímetro mais próximo do marcador móvel, aceitando-se erro de $0,5 \mathrm{~cm}$. As medições foram realizadas em duplicata. Uma terceira medida era realizada quando existia diferença maior que a variação permitida entre as duas primeiras medidas e 
a média entre as duas medidas mais próximas era adotada como medida final ${ }^{24}$.

O IMC (índice de massa corporal) segundo a idade foi adotado para avaliação do estado antropométrico, tomando-se como padrão de referência os pontos de corte preconizados pela OMS: eutrofia, quando situado entre os percentis 3 e 85; magreza, quando abaixo do percentil 3; sobrepeso/obesidade, quando acima do percentil $85^{25}$.

A avaliação do estadiamento puberal foi realizada por autorretratação das características das mamas, idade da ocorrência da menarca e dos pelos púbicos nas meninas, e dos genitais e pelos púbicos nos meninos, utilizando-se gravuras que reproduzem diferentes estágios do estadiamento puberal26,27.

Para o sexo feminino, o início da pubescência foi classificado com base no estágio II do desenvolvimento da mama e o final (pós-pubescência), pela idade da menarca. Para o sexo masculino, o início do estirão (pubescência) foi indicado pelo estágio III de Tanner e o final (pós-pubescência), pelo estágio IV do desenvolvimento da genitália ${ }^{27}$.

O índice de condições ambientais e de moradia foi construído a partir de informações sobre saneamento ambiental, incluindo: abastecimento de água, esgotamento sanitário, coleta pública de lixo; condições de moradia; condições socioeconômicas (renda e escolaridade materna). Atribuiu-se pontuação 0 para a situação mais favorável e 1 para a mais desfavorável. O somatório desses valores classificou o indicador das condições ambientais e de moradia em dois estratos: adequado (escore $\leq 4$ ) e inadequado (escore $>4)^{28}$.

Para avaliar o consumo alimentar, utilizou-se Recordatório Alimentar de 24 Horas $^{29}$. Para aumentar a confiabilidade da informação, utilizou-se álbum com fotos de utensílios e alimentos. Os aspectos do consumo avaliados neste estudo foram a ingestão de balas em substituição às refeições e o consumo de frutas e de outros vegetais.

O nível de atividade física foi adotado como expressão do estilo de vida do escolar e foi avaliado por meio do Questionário de Atividades Físicas Realizadas Ontem (QUAFIRO) ${ }^{30}$. O escore final do QUAFIRO é obtido multiplicando o coeficiente da intensidade da atividade realizada (3,2 ou 1 para intensa, moderada ou leve, respectivamente) pelo coeficiente arbitrário da duração (1, 2 e 3 para < 15, 15-30 e > 30 minutos, respectivamente). A partir da identificação do nível de atividade física realizada, o escolar foi classificado como inativo ( $\leq 5$ pontos) ou ativo ( $>5$ pontos).

A variável dependente ou resposta deste estudo é representada pelos sintomas de transtornos alimentares, estratificada em ausência (0) e presença (1).

Consideraram-se como variáveis independentes ou de exposição: idade (variável contínua), sexo [masculino (0); feminino (1)], escolaridade materna [ $2^{\circ}$ grau completo ou mais (0); Dammy $-1^{\text {a }}$ até $4^{\mathrm{a}}$ série (1); Dammy 2 - 5a a $8^{\mathrm{a}}$ série (2)], estágio puberal [pré-púbere (0); púbere ou pós-púbere (1)], renda familiar [< 1 salário-mínimo (0); $\geq 1$ salário-mínimo (1)], índice ambiental e de moradia [ $>4$ - inadequado $(0) ; \leq 4$ - adequado (1)], estado antropométrico [eutrofia (0); Dammy 1 - magreza (1); Dammy 2 - sobrepeso/obesidade (2)], prática de atividade física [inativo (0); ativo (1)], insatisfação com a imagem corporal [ausência (0); presença (1)], problema de comportamento [ausência (0); presença (1)], problemas de internalização [ausência (0); presença (1)]; problemas de externalização [ausência (0); presença (1)]; consumo de balas e outros doces substituindo refeições [não (0), sim (1)]; consumo de frutas e outros vegetais [não (0); sim (1)].

As características da população foram analisadas por meio da análise descritiva utilizando-se prevalência para os dados categorizados e média e desvio-padrão para variáveis contínuas. Os testes do qui-quadrado e diferença de média foram adotados para avaliar a significância da associação observada.

Empregou-se regressão de Poisson com variância robusta para estimar as razões de prevalências entre os fatores associados e os sintomas de transtornos alimentares. A Razão de Prevalência (RP) e seus intervalos de confiança de 95\% (IC95\%) foram usados, respectivamente, para avaliar a associação e a força da associação entre os eventos investigados ${ }^{31}$.

O processo de modelagem baseou-se na estratégia ordenada em duas etapas. Na primeira, foram realizadas análises bivariadas e selecionadas as variáveis que apresentaram $p$-valor $\leq 0,25^{32}$. Na segunda, realizou-se análise multivariada, com as variáveis pré-selecionadas na análise bivariada. Permaneceram no modelo as variáveis cujos valores de $p$ foram menores do que 0,05 , sendo excluídas sucessivamente do modelo variáveis que, na presença das demais, apresentavam $p$ valores altos, até a construção do modelo final, composto exclusivamente com variáveis cujos valores de $p$ fossem menores do que 0,05 .

Os questionários foram revisados para avaliar a consistência das informações coletadas e correção dos erros porventura decorrentes da codificação. Medidas discrepantes foram identificadas pela análise descritiva dos dados. O banco de dados foi construído utilizando-se o Epi-Info versão 6.0.

Considerando que foram adotados os testes EAT e BITE para avaliar a presença de transtornos alimentares em indivíduos de 12 anos ou mais e somente o EBBIT para os menores de 12 anos, optou-se por construir uma nova variável que refletisse o evento nas duas faixas de idade; essa variável foi estratificada em "sim" (presença de sintomas de transtornos alimentares) e "não" (ausência do evento).

A análise das respostas ao $C B C L$ foi realizada a partir do software Assessment Data Manager (ADM), programa desenvolvido para correção dos escores do $\mathrm{CBCL}^{22}$.

As análises estatísticas foram efetuadas mediante o pacote estatístico STATA (Stata Corp., College Station, Estados Unidos), versão 8.2. O delineamento complexo da amostra foi levado em conta nas análises estatísticas pelo conjunto de comandos SVY do STATA, utilizando-se os pesos associados a cada conglomerado. 


\section{RESULTADOS}

Participaram do estudo 365 escolares, 157 pertencentes a escolas municipais (43\%) e 208, a estaduais (57\%). As características demográficas, biológicas, antropométricas, socioeconômicas e comportamentais dos investigados encontram-se na tabela 1. A população deste estudo foi constituída a partir de uma subamostra de outra investigação, mas não foi observada diferença estatisticamente significante entre as características demográficas da amostra original e a subamostra utilizada nesse estudo (dados não apresentados em tabela).

A amostra é composta predominantemente de escolares de 10 anos ou mais $(87,9 \%)$ e daqueles na fase púbere/pós-púbere do estágio puberal (79,6\%). O percentual de participação das meninas na amostra é similar ao identificado para os meninos (Tabela 1).

A prevalência geral de sintomas de transtornos alimentares identificada neste estudo foi de $23,0 \%$. Segundo a avaliação do EBBIT, a prevalência dos transtornos alimentares entre os escolares de até 12 anos de idade foi de $6,0 \%$. Prevalência mais elevada $(25,1 \%)$ foi identificada entre aqueles com 12 anos ou mais para o BITE. A avaliação produzida pelo EAT-26 indicou prevalência de 1,3\% de sintomas de anorexia nervosa (Tabela 2).

Os resultados das análises de regressão bivariadas de Poisson indicaram que idade ( $\mathrm{RP}_{\text {bruta: }}$ : 1,27; IC95\%: 1,13-1,43), insatisfação com a imagem corporal $\left(\mathrm{RP}_{\text {bruta: }}\right.$ 3,30; IC95\%: 2,10-5,19), consumo de balas em substituição às refeições $\left(\mathrm{RP}_{\text {bruta }}: 2,31 ; \mathrm{IC} 95 \%: 1,32-4,04\right)$ e consumo de frutas e outros vegetais ( $\left.\mathrm{RP}_{\text {bruta }}: 1,83 ; \mathrm{IC} 95 \%: 1,12-2,97\right)$ se associaram positiva e significantemente com a ocorrência de sintomas de transtornos alimentares (Tabela 3).

Não se observou associação estatisticamente significante entre as demais variáveis adotadas neste estudo e a ocorrência de sintomas de transtornos alimentares.

Observou-se que a idade se associou positivamente com a ocorrência de sintomas de transtornos alimentares (RP = 1,25; IC95\%: 1,11-1,40). A presença de insatisfação com a imagem corporal elevou em 4,23 (IC95\%: 2,53-7,08) vezes a prevalência de sintomas de transtornos alimentares no escolar, quando comparado com a ausência de tal insatisfação (Tabela 4).

Os resultados indicaram, ainda, que problemas de comportamento que caracterizam a internalização aumentavam em 1,78 (IC95\%: 1,11-2,85) vez a prevalência de sintomas de transtornos alimentares nos escolares. Outro fator que se revelou estatisticamente associado à ocorrência desses sintomas foi a substituição de grandes refeições por balas (RP: 2,14; IC95\%: 1,24-3,69). Observou-se também que o consumo de frutas e verduras elevou em 2,49 (IC95\%: 1,55-3,99) vezes a prevalência de sintomas de transtornos alimentares nos escolares.
Tabela 1. Características demográficas, biológicas, antropométricas, socioeconômicas e comportamentais das crianças e adolescentes do ensino fundamental da rede pública de ensino - Salvador, Bahia, Brasil, 2008

\begin{tabular}{|c|c|c|}
\hline Variáveis associadas ao indivíduo & $n$ & $\%$ \\
\hline \multicolumn{3}{|l|}{ Sexo } \\
\hline Feminino & 183 & 50,1 \\
\hline Masculino & 182 & 49,9 \\
\hline \multicolumn{3}{|l|}{ Idade } \\
\hline$<10$ anos & 44 & 12,1 \\
\hline$\geq 10$ anos & 321 & 87,9 \\
\hline \multicolumn{3}{|l|}{ Estágio puberal* } \\
\hline Pré-púbere & 74 & 20,3 \\
\hline Púbere/Pós-púbere & 290 & 79,6 \\
\hline \multicolumn{3}{|l|}{ Estado antropométrico } \\
\hline Magreza & 41 & 11,2 \\
\hline Eutrofia & 265 & 72,6 \\
\hline Sobrepeso/0besidade & 59 & 16,2 \\
\hline \multicolumn{3}{|l|}{ Prática de atividade física* } \\
\hline Ativo & 176 & 63,1 \\
\hline Inativo & 103 & 36,9 \\
\hline \multicolumn{3}{|c|}{ Insatisfação com a imagem corporal } \\
\hline Presença & 30 & 8,2 \\
\hline \multicolumn{3}{|c|}{ Problema de comportamento - total* } \\
\hline Presença & 82 & 24,2 \\
\hline \multicolumn{3}{|l|}{ Problemas de internalização* } \\
\hline Presença & 102 & 30,1 \\
\hline \multicolumn{3}{|l|}{ Problemas de externalização* } \\
\hline Presença & 76 & 22,4 \\
\hline \multicolumn{3}{|c|}{ Consumo de balas em substituição às refeições } \\
\hline Sim & 43 & 11,8 \\
\hline \multicolumn{3}{|c|}{ Consumo de frutas e outros vegetais } \\
\hline Sim & 158 & 43,3 \\
\hline \multicolumn{3}{|l|}{ Escolaridade materna* } \\
\hline Até $4^{\text {a }}$ serie & 109 & 30,6 \\
\hline 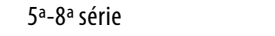 & 134 & 37,5 \\
\hline Ensino médio ou superior & 114 & 31,9 \\
\hline \multicolumn{3}{|l|}{ Renda familiar*/**} \\
\hline$<1 \mathrm{SM}$ & 102 & 28,4 \\
\hline$\geq 1 S M$ & 257 & 71,6 \\
\hline \multicolumn{3}{|l|}{ Índice ambiental e de moradia* } \\
\hline Adequado & 223 & 61,6 \\
\hline Inadequado & 139 & 38,4 \\
\hline
\end{tabular}

*Informações faltantes ** SM: salário-mínimo em dezembro de $2007=R \$ 380,00 ; N=365$.

Tabela 2. Prevalência de sintomas de transtornos alimentares segundo instrumentos de avaliação - Salvador, Bahia, Brasil, 2008

\begin{tabular}{|c|c|c|}
\hline Variáveis & N & $\%$ \\
\hline \multicolumn{3}{|l|}{$\mathrm{EAT}^{\mathrm{a}}$} \\
\hline Presença de sintomas de anorexia nervosa & 3 & 1,3 \\
\hline Ausência & 228 & 98,7 \\
\hline \multicolumn{3}{|l|}{ BITEa $^{a}$} \\
\hline Presença de sintomas de bulimia nervosa & 58 & 25,1 \\
\hline Ausência & 173 & 74,9 \\
\hline \multicolumn{3}{|l|}{$\mathrm{EBBIT}^{\mathrm{b}}$} \\
\hline Presença de sintomas de transtornos alimentares & 8 & 6,0 \\
\hline Ausência & 126 & 94,0 \\
\hline \multicolumn{3}{|l|}{ Sintomas de transtornos alimentares } \\
\hline Presença & 84 & 23,0 \\
\hline Ausência & 281 & 77,0 \\
\hline
\end{tabular}


Tabela 3. Razão de prevalência bruta (RP bruta) das variáveis demográficas, biológicas, antropométricas, socioeconômicas e sintomas de transtornos alimentares em crianças e adolescentes de 7 a 14 anos de idade, da rede pública de ensino em Salvador, Bahia, Brasil, 2008

\begin{tabular}{|c|c|c|c|c|c|}
\hline Variáveis associadas ao indivíduo & $N / \mu$ & \% transtornos & $\mathrm{RP}$ & IC $95 \%$ & $P$ \\
\hline Idade em anos (contínua) & $12,86( \pm 2,18)$ & - & 1,27 & $1,13-1,43$ & $<0,001$ \\
\hline \multicolumn{6}{|l|}{ Sexo } \\
\hline Feminino & 183 & 26,2 & 1,22 & $0,74-2,03$ & 0,429 \\
\hline Masculino & 182 & 19,8 & 1 & & \\
\hline \multicolumn{6}{|l|}{ Estágio puberal } \\
\hline Púbere e pós-púbere & 290 & 27,2 & 3,09 & $0,88-10,78$ & 0,077 \\
\hline Pré-púbere & 74 & 6,8 & 1 & & \\
\hline \multicolumn{6}{|l|}{ Estado antropométrico } \\
\hline Magreza & 41 & 17,1 & 1,20 & $0,49-2,94$ & 0,682 \\
\hline Sobrepeso/Obesidade & 59 & 30,5 & 1,57 & $0,90-2,75$ & 0,111 \\
\hline Eutrofia & 265 & 22,3 & 1 & & \\
\hline \multicolumn{6}{|l|}{ Prática de atividade física* } \\
\hline Ativo & 176 & 26,7 & 1,43 & $0,88-2,33$ & 0,145 \\
\hline Inativo & 103 & 31,1 & 1 & & \\
\hline \multicolumn{6}{|c|}{ Insatisfação com a imagem corporal } \\
\hline Presença & 30 & 76,5 & 3,30 & $2,10-5,19$ & $<0,001$ \\
\hline Ausência & 335 & 29,4 & 1 & & \\
\hline \multicolumn{6}{|c|}{ Problema de comportamento - total ${ }^{*}$} \\
\hline Presença & 82 & 25,6 & 1,24 & $0,72-2,15$ & 0,442 \\
\hline Ausência & 257 & 22,2 & 1 & & \\
\hline \multicolumn{6}{|l|}{ Problemas de internalização* } \\
\hline Presença & 102 & 29,4 & 1,41 & $0,85-2,34$ & 0,184 \\
\hline Ausência & 237 & 20,3 & 1 & & \\
\hline \multicolumn{6}{|l|}{ Problemas de externalização* } \\
\hline Presença & 76 & 18,4 & 0,90 & $0,48-1,68$ & 0,741 \\
\hline Ausência & 263 & 24,3 & 1 & & \\
\hline \multicolumn{6}{|c|}{ Substituição de refeições por balas } \\
\hline Sim & 43 & 37,2 & 2,31 & $1,32-4,04$ & 0,003 \\
\hline Não & 322 & 21,1 & 1 & & \\
\hline \multicolumn{6}{|c|}{ Consumo de frutas e outros vegetais } \\
\hline Sim & 158 & 29,1 & 1,83 & $1,12-2,97$ & 0,015 \\
\hline Não & 207 & 18,4 & 1 & & \\
\hline \multicolumn{6}{|l|}{ Escolaridade materna* } \\
\hline Até a 4a série & 109 & 26,6 & 1,43 & $0,74-2,75$ & 0,281 \\
\hline $5^{\mathrm{a}}$ a $8^{\mathrm{a}}$ série & 134 & 21,6 & 1,53 & $0,81-2,90$ & 0,191 \\
\hline Ensino médio ou superior & 114 & 19,3 & 1 & & \\
\hline \multicolumn{6}{|l|}{ Renda familiar* } \\
\hline$\geq 1 S M$ & 257 & 20,6 & 0,87 & $0,51-1,48$ & 0,609 \\
\hline$<1 S M$ & 102 & 30,4 & 1 & & \\
\hline \multicolumn{6}{|l|}{ Índice ambiental e de moradia* } \\
\hline Adequado & 223 & 25,1 & 0,98 & $0,59-1,63$ & 0,955 \\
\hline Inadequado & 139 & 20,1 & 1 & & \\
\hline
\end{tabular}

* Informaçōes faltantes; ** SM: salário-mínimo em dezembro de $2007=R \$ 380,00 ; \mathrm{N}=365$.

Neste estudo apenas escolaridade materna em níveis intermediários, situada entre a $5^{\mathrm{a}}$ e a $8^{\mathrm{a}}$ série, aumentou em 1,95 (IC95\%: 1,06-3,58) vez a prevalência de tais sintomas, quando considerada como referência a escolaridade mais alta (Tabela 4).

\section{DISCUSSÃO}

A prevalência de sintomas de transtornos alimentares identificada entre os escolares da rede pública de ensino de
Salvador pode ser considerada uma das maiores entre as registradas na literatura nacional (23,0\%). Resultados de estudos pontuais desenvolvidos no Brasil indicam prevalência de sintomas de transtornos alimentares de 13,3\% a 30,2\%33,34; a distribuição dos sintomas de anorexia nervosa variou de $8,3 \%$ a $21,1 \% 2,6,35$ e a de bulimia nervosa, de 1,1\% a 14,0\%3,33.

É possível que essa variação resulte de diferentes métodos de coleta e análise estatística dos dados.

Neste estudo, o EAT detectou a menor prevalência de sintomas de transtorno alimentar (1,3\%), indicando menor ocorrência de sintomas de anorexia nervosa, e o teste 
Tabela 4. Razão de prevalência ajustada das variáveis demográficas, biológicas, antropométricas, socioeconômicas e comportamentais segundo os sintomas de transtornos alimentares em crianças e adolescentes de 7 a 14 anos de idade, da rede pública de ensino em Salvador, Bahia, Brasil, 2008

\begin{tabular}{|c|c|c|c|}
\hline \multirow[t]{2}{*}{ Variáveis } & \multicolumn{3}{|c|}{ RP ajustado } \\
\hline & $\begin{array}{l}\text { Sintomas de } \\
\text { transtornos } \\
\text { alimentares }\end{array}$ & IC95\% & $P$ \\
\hline Idade dos escolares & 1,25 & $1,11-1,40$ & $<0,001$ \\
\hline \multicolumn{4}{|c|}{ Insatisfação com a imagem corporal } \\
\hline Presença & 4,23 & $2,53-7,08$ & $<0,001$ \\
\hline Ausência & - & - & \\
\hline \multicolumn{4}{|l|}{ Problemas de internalização } \\
\hline Presença & 1,78 & $1,11-2,85$ & 0,017 \\
\hline Ausência & - & - & \\
\hline \multicolumn{4}{|c|}{ Consumo de balas em substituição às refeições } \\
\hline $\operatorname{Sim}$ & 2,14 & $1,24-3,69$ & 0,007 \\
\hline Não & - & - & \\
\hline \multicolumn{4}{|c|}{ Consumo de frutas e outros vegetais } \\
\hline $\operatorname{Sim}$ & 2,49 & $1,55-3,99$ & $<0,001$ \\
\hline Não & - & - & \\
\hline \multicolumn{4}{|l|}{ Escolaridade materna* } \\
\hline Até a 4a série & 1,67 & $0,90-3,10$ & 0,103 \\
\hline $5^{\mathrm{a}}$ a $8^{\mathrm{a}}$ série & 1,95 & $1,06-3,58$ & 0,032 \\
\hline Ensino médio ou superior & - & - & \\
\hline
\end{tabular}

BITE, detectando sintomas de bulimia nervosa, apresentou a maior prevalência $(25,1 \%)$ entre os sintomas de transtornos alimentares investigados (Tabela 2).

Indivíduos com transtorno alimentar característico de bulimia, apesar do evidente culto ao corpo, não são exageradamente magros, mas costumam manter o peso normal ou até um leve sobrepeso ${ }^{3}$.

Isso condiz com o fato de que Salvador, embora seja uma cidade litorânea e submetida a altas temperaturas climáticas a maior parte do ano, associando-se com maior exposição corpórea, não se caracteriza por apelo exacerbado do culto à magreza. Evidencia-se a busca pela exposição de corpos bem torneados, "sarados", que expressam sensualidade, mas não necessariamente magros ${ }^{36}$

A ocorrência dos transtornos alimentares tende a aumentar com a idade e parece se associar com maior predisposição à insatisfação corporal, que resulta, especialmente na adolescência, em alterações no comportamento alimentar ${ }^{5}$.

As mudanças biológicas, físicas, psíquicas, aliadas ao desenvolvimento da formação da identidade e do senso de independência, associados ao forte apelo sociocultural do culto à magreza, e encontrando ressonância nos fatores de risco psicológicos individuais e familiares, podem predispor o adolescente aos transtornos alimentares ${ }^{6}$.

Cabe mencionar que, embora o registro dos transtornos alimentares seja mais frequente para adolescentes, informações sobre esse evento são também disponíveis para crianças mais jovens?.
Neste estudo não se observou associação entre sintomas de transtorno alimentar segundo o sexo, sugerindo que esse evento acomete meninos e meninas com igual intensidade; constitui-se, portanto, em um achado de significado epidemiológico, indicando que os meninos também constituem grupos importantes para as medidas de prevenção e controle dessa morbidade. É reconhecido que resultados de vários estudos registram essa relação somente para as meninas ${ }^{8,9,33}$.

Outro elemento que norteia a discussão sobre o comportamento dos portadores de transtornos alimentares diz respeito à prática alimentar. No presente estudo, observou-se que os escolares com sintomas de transtornos alimentares consumiram 2,14 vezes mais balas em substituição às refeições do que aqueles em que tais transtornos estavam ausentes.

Acredita-se que essa estratégia seja adotada como artifício para ingerir alimento de baixa caloria, sabidamente com baixo poder de acumular reservas corporais, em substituição a uma refeição de mais alto teor calórico ${ }^{6}$. Não passa despercebido, também, que essa associação seja fruto da interação entre variáveis que não foram exploradas no desenho analítico deste estudo. Outros estudos, com desenho e proposta analítica mais apropriados, poderiam avaliar tal associação.

Neste estudo, o consumo total de frutas e outros vegetais se associou positivamente com os sintomas de transtornos alimentares (Tabela 4). No entanto, não se pretende levantar a suposição de que o consumo de alimentos desses grupos possa estar diretamente associado com esses transtornos. Nesse sentido, identificou-se que o consumo de frutas e vegetais pelos portadores de sintomas de transtornos alimentares foi maior $(140,10 \mathrm{~g})$ do que a ingestão dos não portadores (126,70 g) (dados não apresentados em tabela).

Esses resultados podem parecer contraditórios, mas encontram explicação na forte tendência de o indivíduo portador de sintomas de transtornos alimentares preferir o consumo de pequenas quantidades de alimentos menos calóricos - entre os quais estão incluídos as frutas, verduras e outros vegetais -, consequentemente induzindo à manutenção ou perda de peso. Tais alimentos são coincidentemente considerados mais saudáveis, pois a tendência de sua escolha por indivíduos com sintomas de transtornos alimentares não possui esse objetivo ${ }^{2,33}$.

Cabe ressaltar que o consumo total desses itens alimentares em ambos os grupos (com e sem transtornos alimentares) não é suficiente para suprir as necessidades nutricionais dos escolares investigados. Assim, o resultado da associação que indica que o consumo de frutas e outros vegetais pelos portadores de sintomas de transtornos alimentares é maior deve ser visto com cautela. Consumo mais elevado de alimentos menos calóricos foi também registrado por Dunker e Philippi ${ }^{6}$ com maior frequência entre alunas portadoras de sintomas de anorexia nervosa do que entre aquelas sem sintomas. Confirmando nossos achados, Nunes e Vasconcelos ${ }^{37}$ verificaram que entre os fatores que determinavam as escolhas alimenta- 
res de adolescentes com transtornos alimentares estavam "a quantidade de calorias ou aquilo que engorda menos".

As evidências indicam, ainda, que a insatisfação com a imagem corporal é um dos principais fatores associados aos sintomas dos transtornos alimentares ${ }^{1}$. Acredita-se que insatisfação corporal gere restrição ao consumo de alimentos, em especial daqueles considerados hipercalóricos, podendo levar os indivíduos a adotarem práticas inadequadas de controle de peso, desde restrição alimentar até uso de condutas purgativas e não purgativas

Identificou-se que a presença de problemas de comportamento de internalização (problemas emocionais como ansiedade, tristeza, depressão, isolamento social e queixas somáticas) explica a prevalência 1,78 vez maior de sintomas de transtornos alimentares do que naqueles em que esses problemas estão ausentes ${ }^{7,8}$.

As evidências científicas indicam que distúrbios como a fobia social e o transtorno obsessivo-compulsivo são mais prevalentes entre os portadores de transtornos alimentares quando comparados com a população sem tais transtornos, sendo caracterizados por frequente fuga de situações sociais por esses indivíduos?.

Estudo caso-controle contabilizou maior ocorrência de sintomas de transtornos ansiosos entre portadoras de distúrbios alimentares, comparadas com controles ${ }^{9}$. Os distúrbios alimentares comprometem aspectos psíquicos fundamentais, afetando a autonomia, ocasionando baixa autoestima e comprometendo habilidades sociais, consequentemente se associando com isolamento social e sintomas depressivos e obsessivos ${ }^{8}$. A relevância dessa associação encontra-se no fato de que a presença de problemas comportamentais ou de personalidade em pacientes com transtornos alimentares está associada a maior gravidade dos transtornos e constitui indicativo de pior prognóstico ${ }^{7}$.

Cabe salientar que este estudo apresenta limitações inerentes aos estudos transversais, particularmente por não contemplar a temporalidade entre os eventos, não permitindo a identificação das relações de causa e efeito.

Reconhece-se, ainda, a delicadeza das questões abordadas neste estudo. Mas o treinamento e o preparo técnico dos profissionais envolvidos na coleta de dados podem ter reduzido o viés de informação que poderia influenciar os resultados.

Salienta-se, ainda, que o cuidado metodológico adotado na coleta dos dados e no desenho estatístico adequado ao estudo pode contribuir para a fidedignidade dos resultados. Assim, mesmo conhecendo as limitações comentadas acima, os resultados deste estudo merecem crédito científico.

\section{CONCLUSÃO}

A alta prevalência de sintomas de transtornos alimentares detectada entre os escolares investigados reforça a necessidade de intervir precocemente nos fatores associados à sua ocorrência, visando ao controle e à prevenção desses eventos.

\section{AGRADECIMENTOS}

À professora Darci Neves dos Santos, pela colaboração técnica e intelectual para a elaboração do artigo científico; à Fundação de Amparo à Pesquisa do Estado da Bahia (Fapesb), pelo auxílio financeiro para execução do projeto.

\section{CONFLITOS DE INTERESSE}

Os autores declaram não possuir conflitos de interesse.

\section{REFERÊNCIAS}

1. American Academy of Pediatrics - AAP (US). Children and adolescents with eating disorders: the state of the art. Pediatrics. 2003;111:1-13.

2. Alves E, Vasconcelos FAG, Calvo MCM, Neves J. Prevalência de sintomas de anorexia nervosa e insatisfação com a imagem corporal em adolescentes do sexo feminino do município de Florianópolis, Santa Catarina, Brasil. Cad Saude Publica. 2008;24(3):503-12.

3. Silva CGC, Andrade CAA, Araújo EC, Cavalcanti AMTS. Riscos e fatores de risco para transtornos alimentares em adolescentes do gênero feminino. Rev Enferm UFPE on line. 2007;1 (2):89-96.

4. Branco LM, Hilario MOE, Cintra IP. Percepção e satisfação corporal em adolescentes e a relação com seu estado nutricional. Rev Psiq Clín. 2006; 33(6):292-6.

5. Triches RM, Giugliani ERJ. Insatisfação corporal em escolares de dois municípios da região Sul do Brasil. Rev Nutr. 2007;20(2):119-28.

6. Dunker KLL, Philippi ST. Hábitos e comportamentos alimentares de adolescentes com sintomas de anorexia nervosa. Rev Nutr. 2003:16(1):51-60.

7. Masjuán MG, Aranda FF, Raich RM. Bulimia nerviosa y trastornos de la personalidad. Una revisión teórica de la literatura. Int J Clin Health Psychol. 2003;3(2):335-49.

8. American Academy of Pediatrics - AAP (US). Practice guideline for the treatment of patients with eating disorders. 2nd ed.; 2000.

9. Fleitlich BW, Larino MA, Cobelo A, Cordas TA. Anorexia nervosa na adolescência. J Pediatr. 2000;76(Supl 3):S323-9.

10. Mellin P, Araújo AM. Transtornos alimentares em homens: um desafio diagnóstico. Rev Bras Psiquiatr. 2002;24(Supl III):S73-6.

11. Dunker KLL, Fernandes CPB, Carreira-Filho D. Influência do nível socioeconômico sobre comportamentos de risco para transtornos alimentares em adolescentes. J Bras Psiquiatr. 2009;58(3):156-61.

12. Borges CQ, Silva RCR, Assis AMO, Pinto EJ, Fiaccone RL, Pinheiro SMC. Fatores associados à anemia em crianças e adolescentes de escolas públicas de Salvador, Bahia, Brasil. Cad Saude Publica. 2009;25(4):877-88.

13. Candy CM, Fee VE. Underlying dimensions and psychometric properties of the eating behaviors and Body Image Test for preadolescent girls. J Clin Child Psychol. 1998:27(1):117-27.

14. Galindo EMC. Tradução, adaptação e validação do Eating Behaviours and Body Image Test (EBBIT) em crianças do sexo feminino na cidade de Ribeirão Preto, São Paulo [dissertação]. Ribeirão Preto: Universidade de São Paulo; 2005

15. Garner DM, Garfinkel PE. The eating attitudes test: psychometric features and clinical correlates. Psychol Med. 1982;12:871-8.

16. Henderson M, Freeman CPL. A self-rating scale for bulimia: the BITE. Br J Psychiatr. 1987;150:18-24 
17. Bighetti F. Tradução e validação do Eating Attitudes Test (EAT-26) em adolescentes do sexo feminino na cidade de Ribeirão Preto - SP [dissertação]. Ribeirão Preto: Escola de Enfermagem de Ribeirão Preto, Universidade de São Paulo; 2003.

18. Cordás B, Athanássios T, Hochgraf P. O "BITE": instrumento para avaliação da bulimia nervosa - versão para o português. J Bras Psiquiatr. 1993;42(3):141-4.

19. Cooper PJ, Taylor MJ, Cooper Z, Fairburn CG. The development and validation of the Body Shape Questionnaire. Int J Eating Disord. 1987;6:485-94.

20. Cordás TA, Castillo S. Imagem corporal nos transtornos alimentares-instrumentos de avaliação: "Body Shape Questionnaire". Psiquiatr Biol. 1994;2(1):17-21.

21. Conti MA. A imagem corporal de adolescentes: validação e reprodutibilidade de instrumentos [tese]. São Paulo: Departamento de Epidemiologia da Faculdade de Saúde Pública, Universidade de São Paulo; 2007.

22. Achenbach TM, Rescorla LA. Manual for the Achenbach of Empirically Based Assessment (ASEBA) School-Ages Forms \& Profiles. Burlington: University of Vermont; 2001.

23. Bordin IAS, Mari JJ, Caeiro MF. Validação da versão brasileira do Child Behavior Checklist (CBCL) (Inventário de Comportamento da Infância e da Adolescência): dados preliminares. Rev ABP-APAL. 1995;17(2):55-66.

24. Lohman $\mathrm{TG}$, Roche AF, Martorell R. Anthropometric standardization reference manual. Abridged edition; Champaign, Illinois, 1988.

25. World Health Organization - WHO. WHO Child Growth Standards: length/height-forage, weight-for-age, weight-for-length, weight-for-height and body mass index-for-age: methods and development. Geneva: World Health Organization; 2007.

26. Marshall WA, Tanner JM. Variations in the pattern of pubertal changes in boys. Arch Dis Child. 1970;45:13-23.
27. Marshall WA, Tanner JM. Variations in pattern of pubertal changes in girls. Arch Dis Child. 1969;44:291-303.

28. Issler RM, Giugliani ER. Identificação de grupos mais vulneráveis à desnutrição infantil pela medição do nível de pobreza. J Pediatr (Rio de Janeiro). 1997;73:101-5.

29. Biró C, Hulshaf K, Ovesen L, Cruz A. Selection of methodology to assess food intake. Eur J Clin Nutr. 2002;56(Suppl 2):S25-32.

30. Suñe FR, Dias-da-Costa JS, Olinto MTA, Pattussi MP. Prevalência e fatores associados para o sobrepeso e obesidade em escolares de uma cidade no Sul do Brasil. Cad Saude Publica. 2007;23(6):1361-71.

31. Coutinho LMS, Scazufca M, Menezes PR. Métodos para estimar razão de prevalência em estudos de corte transversal. Rev Saude Publica. 2008;42(6):992-8.

32. Hosmer DW, Lemeshow S. Applied Logistic Regression. A Wiley-Interscience publication. New York: Jonh Wiley \& Sons; 1989.

33. Vilela JEM, Lamounier JA, Dellaretti-Filho MA, Barros-Neto JR, Horta GM. Transtornos alimentares em escolares. J Pediatr (Rio de Janeiro). 2004;80(1):49-54.

34. Nunes MA, Olinto MTA, Barros FC, Camey S. Influência da percepção do peso e do índice de massa corporal nos comportamentos alimentares anormais. Rev Bras Psiquiatr. 2001;23(1):21-7.

35. Chiodini JS, Oliveira MRM. Comportamento alimentar de adolescentes: aplicação do EAT26 em uma escola pública. Saude Rev. 2003;5(9):53-8.

36. Iriart JAB, Chaves JC, Orleans RG. Culto ao corpo e uso de anabolizantes entre praticantes de musculação. Cad Saude Pública. 2009;25(4):773-82.

37. Nunes AL, Vasconcelos FA. [Eating disorders in the view of teenage girls from Florianópolis, Santa Catarina State: a phenomenological approach]. Ciênc Saúde Coletiva. 2010;15(2):539-50 